\title{
In vivo evidence for a significant role of folylpolyglutamate synthase in combined chemotherapy with oral fluoropyrimidine, UFT or S-1, and leucovorin
}

\author{
SAYAKA TSUKIOKA, ETSUKO SAKAMOTO, HIROAKI TSUJIMOTO, FUMIO NAKAGAWA, \\ HITOSHI SAITO, JUNJI UCHIDA, MAMORU KINIWA and MASAKAZU FUKUSHIMA \\ Tokushima Research Center, Taiho Pharmaceutical Co., Ltd, \\ 224-2 Hiraishi-ebisuno Kawauchi-cho, Tokushima 771-0194, Japan
}

Received November 24, 2010; Accepted January 14, 2011

DOI: $10.3892 /$ or.2011.1206

\begin{abstract}
Combined chemotherapy with 5-fluorouracil and leucovorin (LV) has been widely used for the treatment of patients with colorectal cancer. Given that LV effects are attributable to increased levels of reduced folate in cancer cells, we attempted here to show the in vivo role of folylpolyglutamate synthetase (FPGS), which stabilizes intracellular reduced folate, in the anticancer activities of oral fluoropyrimidines, UFT or S-1, combined with LV. To this end, HCT-15 human colon cancer cells were knocked down for FPGS expression by RNA interference. The cell line stably expressing FPGS shRNA (FPGS shRNA HCT-15) was cloned and transferred subcutaneously into nude mice fed a low-folate diet. FPGS shRNA HCT-15 tumors expressed a significantly lower level of FPGS at protein and mRNA levels than parental HCT-15 cells, and the levels of reduced folate in FPGS shRNA HCT-15 tumors became $57 \%$ of those in parent after a single administration of $10 \mathrm{mg} / \mathrm{kg}$ of LV. Notably, FPGS downregulation did not affect the tumor growth or sensitivity to fluoropyrimidine. Importantly, we observed that LV given for 14 days failed to enhance the anticancer effects of UFT and S-1 in FPGS shRNA HCT-15. This was in keeping with the results that LV did not increase the ternary complex of TS, FdUMP and reduced folate. In conclusion, the present results provide in vivo evidence that intratumor FPGS plays an important role in the efficacy of oral fluoropyrimidine plus LV therapy for colorectal cancer.
\end{abstract}

\section{Introduction}

Combined chemotherapy with 5-fluorouracil (5-FU) and leucovorin (LV) has been widely used to treat patients

Correspondence to: Dr Sayaka Tsukioka, Tokushima Research Center, Taiho Pharmaceutical Co., Ltd, 224-2 Hiraishi-ebisuno Kawauchi-cho, Tokushima 771-0194, Japan

E-mail: s-tsukioka@taiho.co.jp

Key words: folylpolyglutamate synthase, 5-fluorouracil, leucovorin, S-1, UFT with colorectal cancer (CRC). In Japan, UFT, oral fluoropyrimidines instead of intravenous 5-FU, with LV (UFT/ $\mathrm{LV}$ ) have been also used based on evidence of equivalent efficacy to 5-FU/LV for the treatment of CRC patients in both advanced and adjuvant settings (1-3). UFT is an orally active combination of tegafur (FT; a prodrug of 5-FU) and uracil [a competitive inhibitor of dihydropyrimidine dehydrogenase (DPD) that degrades 5-FU] in a 1:4 molar ratio. In addition, $\mathrm{S}-1$ is an orally active combined formulation of FT, gimeracil (CDHP; a potent inhibitor of DPD), and oteracil [Oxo; an inhibitor of orotate phosphoribosyltransferase (OPRT) that phosphorylates 5-FU in the gastrointestinal tract, which thereby reduces its toxic gastrointestinal effects] at molar ratios of 1:0.4:1 (4). The efficacy of S-1 for the treatment of metastatic CRC has been demonstrated in clinical studies (5), and phase II clinical trials of S-1/LV combination therapy for $\mathrm{CRC}$ are in progress in Asia.

Fluoropyrimidine derivatives have been shown to exert anticancer effects primarily through the inhibition of thymidylate synthase (TS) by forming covalent ternary complexes with FdUMP and a representative reduced folate, 5,10-methylenetetrahydrofolate $\left(\mathrm{CH}_{2} \mathrm{FH}_{4}\right)$. Although $\mathrm{LV}$ itself has no antitumor activity, LV enhances the anticancer effect of 5-FU by increasing the intratumor levels of reduced folate, which resultantly stabilizing the ternary complex (6).

Many studies have discussed the factors that determine the clinical efficacy of 5-FU-based chemotherapy (6-9). However, limited information is still available with regard to the determinant of the LV effect. It is note that orally or intravenously administrated LV is metabolized to monoglutamate $5-\mathrm{CH}_{3} \mathrm{FH}_{4}$, circulating form in blood, which is transported across the cell membrane. Once taken up into cells, intracellular folates are converted to polyglutamates by folylpolyglutamyl synthetase (FPGS), and polyglutamated folates are better retained in cells and more stabilize the ternary complex more than monoglutamate folates (10). FPGS has been shown to be one of the determinants of intracellular reduced folate level after $\mathrm{LV}$ treatment in vitro (11) and in clinical studies (12). However, it remains unclear in vivo or in clinical studies whether the FPGS status in the tumors affects the ability of LV to enhance the antitumor 
activity of fluoropyrimidine. Thus, using short hairpin RNA (shRNA) to downregulate FPGS, we assessed the impact of FPGS expression levels on LV-mediated enhancement of the intratumor reduced folate and the antitumor effects of UFT or $\mathrm{S}-1$ in a mouse xenograft model.

\section{Materials and methods}

Materials. The FT, CDHP, Oxo, Uracil and LV used in this study were provided by Taiho Pharmaceutical Co., Ltd. (Tokyo, Japan). Hydroxypropylmethylcellulose (HPMC) was purchased from Shin-Etsu Chemical Co., Ltd. (Tokyo, Japan). $\left[6-{ }^{3} \mathrm{H}\right]-\mathrm{FdUMP}$ and $\left[6-{ }^{14} \mathrm{C}\right]-5-\mathrm{FU}$ were purchased from Moravek Biochemicals Inc. (Brea, CA, USA).

Cell lines and animals. The human colon cancer cell line HCT-15 was obtained from Dainippon Pharma Co., Ltd. (Osaka, Japan) and was grown in RPMI-1640 medium (Sigma Aldrich Japan, Tokyo, Japan) with $10 \%$ fetal bovine serum (MP Biomedicals, Inc., Aurora, OH, USA). Male BALB/cA-nu/nu mice (5-week-old) were purchased from Charles River Japan Inc. (Tokyo, Japan). They were fed a low-folate pellet diet (AIN-93M-based Folate-Deficient Rodent Diet, Oriental Yeast Co., Ltd., Tokyo, Japan.) with filtered water ad libitum. The low-folate diet contained $10 \%$ of the folate of the normal diet. The mice were kept in laminar air-flow units throughout the experiments, all of which were performed in compliance with the regulations of the Animal Experimentation Committee of Taiho Pharmaceutical Co., Ltd.

Generation of colon cancer cell lines stably expressing FPGS shRNA. The shRNA oligonucleotides for FPGS were purchased from Invitrogen (Gaithersburg, MD, USA). The shRNA oligonucleotides were subcloned into pBAsi-hU6Neo vector (Takara, Shiga, Japan) and transfected into HCT-15 cells using Lipofectamine 2000 (Invitrogen). These cells were incubated with $700 \mu \mathrm{g} / \mathrm{ml}$ of G418 (Gibco, Tokyo, Japan) to select for cells transfected with the construct. We screened clones of HCT-15 cells expressing FPGS shRNA by quantitative real-time RT-PCR. Total RNA was extracted using the RNeasy mini kit (Qiagen, Mississauga, Canada) and then subjected to reverse transcription. Quantitative real-time RT-PCR was performed on a PRISM 7900 sequence detector (Applied Biosystems, Foster City, CA, USA) using a TaqMan Universal PCR Master mix (Applied Biosystems). The geneexpression levels were normalized to $\beta$-actin. The primers and TaqMan probes were prepared using Assay-on-Demand gene-expression products (Applied Biosystems).

Drug preparation and administration. UFT was prepared by mixing FT and Uracil at molar ratios of 1:4 in 0.5\% HPMC solution. S-1 was prepared by mixing FT, CDHP and Oxo at molar ratios of 1:0.4:1 in 0.5\% HPMC solution. The dosages of UFT or S-1 were expressed in terms of their contents of FT. LV was suspended in 0.5\% HPMC solution. S-1 $(8.3 \mathrm{mg} / \mathrm{kg} /$ day), UFT (16.7 mg/kg/day) and LV (10 mg/kg/day) were orally administered for 14 days.

Antitumor experiments. Human tumor xenografts were prepared by subcutaneous implantation of a tumor fragment
( $\sim 2 \mathrm{~mm}$ in diameter) into the right side of the back of each nude mouse (at $\sim 7$-week-old). On 100-200 $\mathrm{mm}^{3}$ tumor volumes (TVs), the animals were randomized to control or treatment groups in such a way as to make the average TVs the same for the groups. The drugs were given for 14 consecutive days. Tumor size and body weight were measured twice weekly throughout the experiments. The TV, relative tumor volume (RTV), tumor growth-inhibition (TGI) rate and body-weight change (BWC) were calculated as follows:

TV $\left(\mathrm{mm}^{3}\right)=$ length $(\mathrm{mm}) \times$ width $(\mathrm{mm}) \times$ width $(\mathrm{mm}) \times 0.5$

$\mathrm{RTV}=(\mathrm{TV}$ on day 15$) /(\mathrm{TV}$ on day 0$)$

TGI $(\%)=[1-($ mean RTV of treatment group $) /($ mean RTV of control group) $] \times 100$

BWC $(\%)=[($ mean body weight at day 15$) /($ mean body weight at day 0$)-1] \times 100$

Determination of TS activity. TS activity was determined as the number of $\left[6-{ }^{3} \mathrm{H}\right]-$ FdUMP binding sites in the $105,000 \mathrm{~g}$ supernatant of tumor tissue homogenates as described by Spears et al (13). An aliquot of the supernatant was used to determine the soluble protein by the method of Bradford. The supernatant was incubated with $\left[6-{ }^{3} \mathrm{H}\right]-\mathrm{FdUMP}$ in the presence of excess $\mathrm{CH}_{2} \mathrm{FH}_{4}$ for $20 \mathrm{~min}$ at $30^{\circ} \mathrm{C}$, and the radioactivity in the acid-insoluble fraction was measured with a liquid scintillation counter (TRI-CARB 2000CA; Packard Instruments, Meriden, CT, USA).

Determination of OPRT activity. Tumors were placed in 3-time volumes of ice-cold $10 \mathrm{mM}$ Tris- $\mathrm{HCl}$ buffer ( $\mathrm{pH} \mathrm{7.4),}$ containing $1 \mathrm{mM}$ EDTA and $0.5 \mathrm{mM}$ DTT and homogenized. OPRT activity was determined using a previously described 5-FU phosphorylation assay (14). The supernatant of tumor tissue homogenates was incubated with $20 \mu \mathrm{M}\left[6-{ }^{14} \mathrm{C}\right] 5-\mathrm{FU}$; $50 \mathrm{mM}$ Tris- $\mathrm{HCl}, \mathrm{pH} 8.0 ; 5 \mathrm{mM} \mathrm{MgCl} ; 10 \mathrm{mM} \mathrm{NaF} ; 0.5 \mathrm{mM}$ DTT and $4 \mathrm{mM}$ phosphoribosylpyrophosphate at $37^{\circ} \mathrm{C}$ for $10 \mathrm{~min}$. The incubation was terminated by the addition of methanol followed by centrifugation at 10000 revolutions per min (rpm) for $5 \mathrm{~min}$. The supernatant was dried up, and dissolved in distilled water $(100 \mu \mathrm{l})$, and $40 \mu \mathrm{l}$ aliquots were applied to the ODS column (Daiso, Osaka, Japan) in HPLC (Shimadzu, Kyoto, Japan). The mobile phase was used as follows: solvent A, acetonitrile; solvent B, $10 \mathrm{mM}$ potassium dihydrogenphosphate buffer. Elution gradient used: the gradient started with $0.5 \% \mathrm{~A}$ at $0-5 \mathrm{~min}, 0.5 \% \mathrm{~A}$ to reach $50 \%$ at $5-9 \mathrm{~min}, 50 \% \mathrm{~A}$ at $9-12 \mathrm{~min}, 50 \%$ A to reach $0.5 \%$ at 12-20 min. The extracts were mixed with the Ultima Flow M (PerkinElmer, Waltham, MA, USA). The radioactivity was measured in a Flow Scintillation Analyzer (PerkinElmer).

Determination of intratumoral reduced folate level. The assay for reduced folate is based on the entrapment of $\mathrm{CH}_{2} \mathrm{FH}_{4}$ into a stable ternary complex with excess recombinant human TS protein and excess [6- $\left.{ }^{3} \mathrm{H}\right]$ FdUMP (15). Since $\mathrm{CH}_{2} \mathrm{FH}_{4}$ can potentially be dissociated into $\mathrm{FH}_{4}$ and formaldehyde under these conditions, the sum of these folates is measured. Tumor tissues were homogenized with 3-time volumes of ice-cold 
A

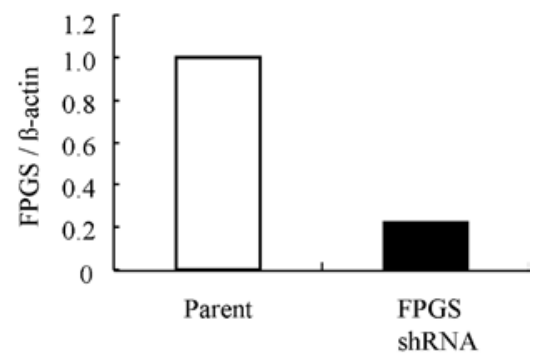

B

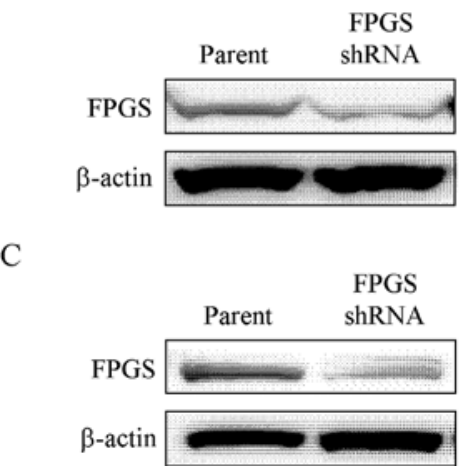

Figure 1. Reduced expression of FPGS in HCT-15 shRNA cells. Parental and HCT-15 shRNA cells cultured in vitro were measured for FPGS expression at mRNA levels by real-time RT-PCR and normalized by $\beta$-actin expression (A), at protein level by Western blotting (B). These cells were transferred into nude mice, and tumor tissues were measured for FPGS expression at protein levels $(\mathrm{C})$. Levels of $\beta$-actin are shown as loading controls and the intensity of FPGS relative to the parent was indicated in (B) and (C).

$10 \mathrm{mM}$ phosphate buffer ( $\mathrm{pH} 7.0$ ), containing $2 \mathrm{mg} / \mathrm{ml}$ ascorbic acid and $40 \mathrm{mM}$ 2-mercaptoethanol. After centrifugation, the supernatant was diluted with homogenizing buffer to $8 \mathrm{mg}$ protein $/ \mathrm{ml}$, then placed immediately in a boiling water bath for $1 \mathrm{~min}$ and centrifuged to remove the precipitated protein. The supernatant was used for folate determination in a reaction mixture containing $5 \mu \mathrm{g}$ recombinant human TS protein, $125 \mathrm{nM}\left[6-{ }^{3} \mathrm{H}\right]-\mathrm{FdUMP}$, and $6.5 \mathrm{mM}$ formaldehyde in $200 \mu \mathrm{l}$ of $50 \mathrm{mM}$ Tris-HCl buffer ( $\mathrm{pH} 7.4)$. The supernatant $(50 \mu \mathrm{l})$ was added to $200 \mu \mathrm{l}$ of the reaction mixture, incubated at $30^{\circ} \mathrm{C}$ for $50 \mathrm{~min}$, and then the radioactivity in the acid-insoluble fraction was measured with a liquid scintillation counter.

Western blotting. Samples of lysates containing $40 \mu \mathrm{g}$ protein were resolved by sodium dodecyl sulfate polyacrylamide gel electrophoresis (SDS-PAGE), transferred to polyvinylidene fluoride (PVDF) membranes, blocked in blocking solution (UK-B80, DS Pharma Biomedical Co., Ltd. Osaka, Japan), and incubated with primary antibodies (anti-human FPGS antibody diluted at 1:1000, as previously reported (11); antihuman TS antibody diluted at 1:5000, Taiho Pharmaceutical; anti-human $\beta$-actin antibody diluted at 1:10000, \#A5441 Sigma, St. Louis, MO, USA) for $1 \mathrm{~h}$ at room temperature. Membranes were washed three times for $10 \mathrm{~min}$ each in TBST (20 mM Tris-HCl, pH 7.5, 150 mM NaCl, 0.05\% Tween-20) and incubated with horseradish peroxidaseconjugated secondary antibodies for $1 \mathrm{~h}$ at room temperature.

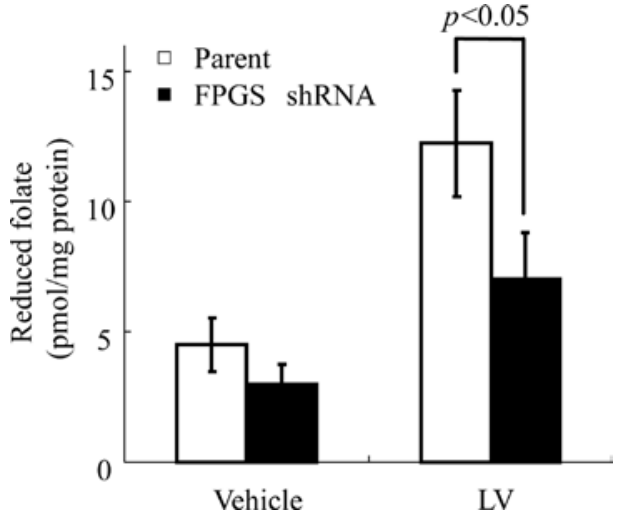

Figure 2. FPGS shRNA interfered with LV-induced increase in intratumoral reduced folate levels. Folate-depleted mice bearing human colon cancer tumors were given orally vehicle (open columns) or a single dose of $10 \mathrm{mg} / \mathrm{kg} \mathrm{LV}$ (closed columns). After $24 \mathrm{~h}$, tumor tissues were isolated and subjected for the measurement of reduced folate. Values are the mean $\pm \mathrm{SD}$ of three mice. Statistical analysis was performed by Student's t-test, $\mathrm{p}<0.05$ was considered to indicate statistically significant difference.

After three 10-min washes, the membranes were incubated with enhanced ECL reagent (\#34096 Thermo, Rockford, IL, USA) for $1 \mathrm{~min}$ and chemiluminescence was detected with LAS 3000 (Fujifilm, Tokyo, Japan).

Statistical analysis. The significance of differences in reduced folate level and RTV were analyzed with Student's t-test and Aspin-Welch's t-test respectively using EXSAS (version 7.11, Arm Co., Ltd., Osaka, Japan). p $<0.05$ was considered to be significant.

\section{Results}

Establishment of colon cancer cell lines stably expressing FPGS shRNA. We first planned to establish the human colon cancer cell lines that express downregulated levels of FPGS but keep the sensitivity to 5-FU in vitro compare to the parental cell lines. Several CRC cell lines were tested if they express significant levels of FPGS and respond to 5-FU in vitro. Selected cell lines were transfected with shRNA oligonucleotide for FPGS, subcloned based on their expression of FPGS shRNA. Cell lines stably expressed FPGS shRNA were subcloned and transferred subcutaneously into nude mice fed low folate diet (folate-deficient mice). Fig. 1 shows characteristic features of a representative cell line, the HCT-15 FPGS shRNA; as compared with parental HCT-15 cells, they expressed low levels of FPGS mRNA and protein in vitro and significantly lower levels of FPGS protein in vivo. Note that the intratumoral activities of TS and OPRT, which were reported to play an important role in the chemosensitivity of cancer cells to 5-FU (6-9), were invariable under FPGS shRNA (Table I).

Effect of FPGS downregulation on reduced folate levels in colon tumor xenografts. We next investigated the significance of FPGS in regulating intratumor reduced folate levels. Fig. 2 shows the levels of reduced folate in the tumors at $24 \mathrm{~h}$ after 

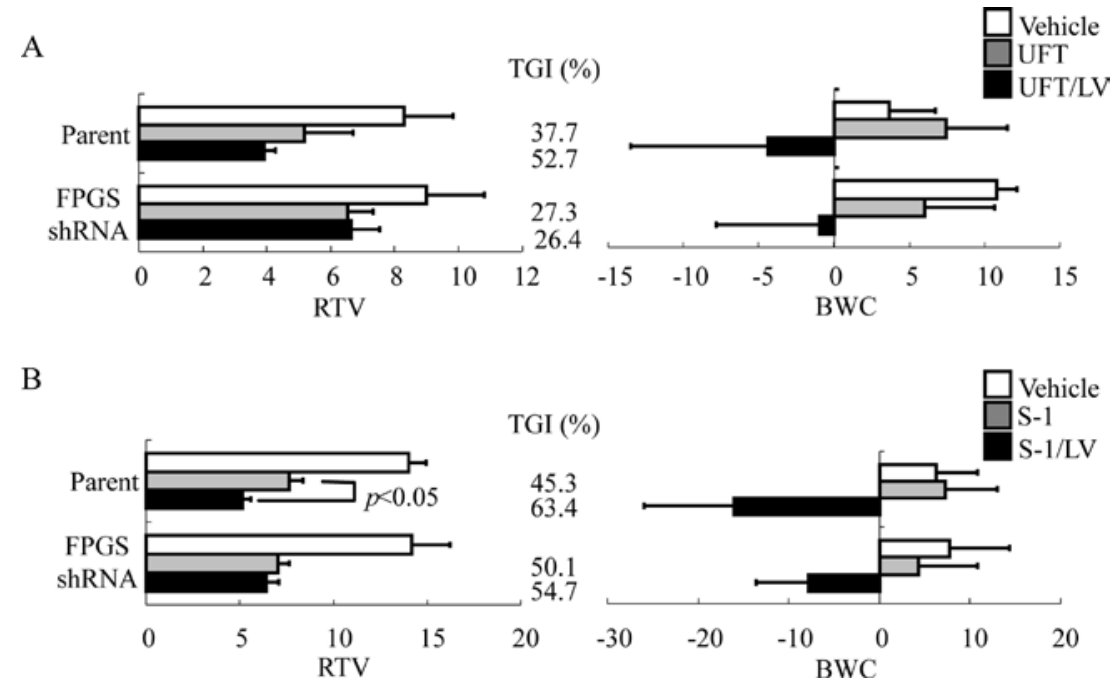

Figure 3. Anticancer effect of UFT/LV or S-1/LV treatment on parental and FPGS shRNA HCT-15 cells. UFT $16.7 \mathrm{mg} / \mathrm{kg} / \mathrm{day}$ (A) or S-1 $8.3 \mathrm{mg} / \mathrm{kg} / \mathrm{day}$ (B) with/without LV (10 mg/kg/day) were given orally for 14 consecutive days. On day 15 , RTV and BWC were evaluated. Values indicate the mean \pm SD of 7 mice. Statistical analysis was performed by Aspin-Welch's t-test, $\mathrm{p}<0.05$ was considered to indicate statistically significant difference.

A

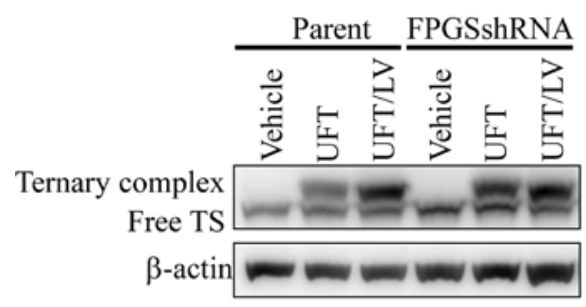

B

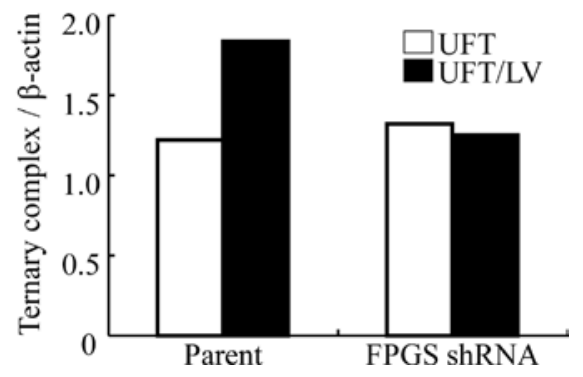

Figure 4. Formation of ternary complexes after UFT or UFT/LV treatment. (A) The protein levels of free TS and ternary complex in pooled parental HCT-15 or FPGS shRNA HCT-15 tumors were detected by Western blotting on day 15 after UFT or UFT in combination with LV treatment. Levels of $\beta$-actin are shown as loading controls. (B) Relative ternary complex level was calibrated as the ratio of the band intensity of the ternary complex to $\beta$-actin.

vehicle or LV treatment. In control group, the levels of reduced folate in FPGS shRNA HCT-15 tumors were $66 \%$ of that in parent tumors. Single administration of LV significantly elevated the reduced folate levels in tumors, while the reduced folate levels in FPGS shRNA HCT-15 tumors became 57\% of that in parent tumors in the LV treatment group $(\mathrm{p}<0.05)$.

Effect of FPGS downregulation on the antitumor effects of UFT or S-1 in combination with LV. Subsequently, we
Table I.Effects of FPGS shRNA on TS and OPRT enzyme activities in tumor tissues of the human colon cancer xenografts. ${ }^{a}$

\begin{tabular}{lcc}
\hline Tumors & $\begin{array}{c}\text { TS } \\
(\mathrm{pmol} / \mathrm{mg} \text { protein })\end{array}$ & $\begin{array}{c}\text { OPRT } \\
(\mathrm{pmol} / \mathrm{min} / \mathrm{mg} \text { protein })\end{array}$ \\
\hline Parent & $0.82 \pm 0.22$ & $7.57 \pm 7.40$ \\
FPGS shRNA & $0.66 \pm 0.01$ & $6.52 \pm 3.70$ \\
\hline
\end{tabular}

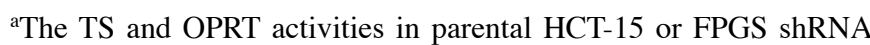
HCT- 15 tumors were measured by the methods described in Materials and methods. TS activity was determined as the number of $\left[6-{ }^{3} \mathrm{H}\right]-$ FdUMP binding sites and OPRT activity was determined using 5-FU as a substrate. Values indicate the mean $\pm \mathrm{SD}$ of 3 mice.

examined whether expression levels of FPGS affected the effectiveness of LV on antitumor activity of oral fluoropyrimidine with LV. UFT/LV were orally administered for 14 consecutive days to nude mice bearing parental HCT-15 or FPGS shRNA HCT-15 tumors $(\mathrm{n}=7)$. The tumor growth of FPGS shRNA HCT-15 was similar to that of parental HCT-15. LV alone had no antitumor activity, while UFT alone or UFT/ LV treatment inhibited tumor growth regardless of FPGS downregulation $(\mathrm{p}<0.05$; Fig. 3A). UFT/LV clearly inhibited parental HCT-15 tumor growth more effectively than UFT alone, however LV co-administration failed to further potentiate the antitumor effect of UFT in FPGS shRNA HCT-15 xenografts (Fig. 3A). On the other hand, LV co-administration resulted in body weight loss in the same manner in both parental HCT-15 and FPGS shRNA HCT-15 bearing mice, although this effect was tolerable. Furthermore, similar results were also observed in the case of the administration of S-1 instead of UFT (Fig. 3B), suggesting that FPGS downregulation suppressed LV-induced enhancement of antitumor effect of UFT or S-1 as a result of the loss of reduced folate induced by LV. 
Effect of FPGS downregulation on the ternary complex formation. To support the data of antitumor activities, we measured the TS ternary complexes, thought to indicate the effect of 5-FU, using Western blotting in parental and FPGS shRNA HCT-15 tumor xenografts $24 \mathrm{~h}$ after treatment with UFT alone or UFT/LV for 14 consecutive days (Fig. 4). LV co-administration increased the ternary complex formation in parental HCT-15 tumors, however, the increase was not observed in FPGS shRNA HCT-15 tumors.

\section{Discussion}

The present study was designed to demonstrate the role of FPGS in potency of oral furuoropyrimidine combined with LV in vivo. To this end, we newly established the human colon cancer cell lines which stably expressed FPGS shRNA. Representative FPGS shRNA HCT-15 cells in culture expressed significantly lower levels of FPGS at mRNA and protein levels compared to parental HCT-15 cells. More importantly, HCT-15 FPGS shRNA tumor tissue isolated from nude mice retained the suppressed levels of FPGS expression at protein levels. It should be noted that FPGS downregulation exhibited no significant impact on the tumor growth or intra-tumoral activities of TS and OPRT, which may affect the efficacy of 5-FU treatment. The results were consistent with the findings reported by Sakamoto et al who showed no significant difference in the $\mathrm{IC}_{50}$ values of fluorodeoxyuridine between control siRNA and FPGS siRNA transfected cells transiently (11).

Based on these findings, it is reasonable to assume that the mouse xenograft model of downregulated FPGS is valid to investigate the role of FPGS in LV therapy in terms of intratumoral reduced folate levels as well as anticancer activities of UFT or S-1 plus LV. Therefore, we compare the changes in the levels of reduced folate in parental and FPGS shRNA HCT-15 cells before and after oral administration of LV. There was no significant difference in reduced folate levels between parental and FPGS shRNA HCT-15 cells, but an increase in intratumoral reduced folate after LV treatment was definitely suppressed in FPGS shRNA HCT-15 cells compared to those in parental cells. The results suggest that FPGS stabilize newly incorporated into cells more effectively than basal one, but further analysis is needed to understand the precise mechanism of intratumoral FPGS. Important is the observation that in contrast to those seen in parental cells, LV treatment failed to enhance the anticancer activity of UFT or S-1 combined with LV, in FPGS shRNA HCT-15 cells. Moreover, LV co-administration formed a much larger ternary complex than UFT alone in parental but not in FPGS shRNA HCT-15 cells. These data are in agreement with previous studies in that the increased levels of intratumoral reduced folate after LV treatment stabilized the ternary complex and potentiated the TS inhibition (16-18).

In conclusion, our study provides first in vivo evidence that intratumoral FPGS status may regulates the LV-enhanced anticancer activity of UFT and S-1. Taken together with the recent study that FPGS expression and activity may correlate with the cytotoxicity of 5-FU with or without LV by in vitro studies $(19,20)$, we propose the requirement of further clinical studies to identify FPGS as a significant enzyme for predicting the efficacy of LV in combination with oral fluoropyrimidine.

\section{Acknowledgments}

We thank Professor J. Patrick Barron, the Department of International Medical Communications of Tokyo Medical University for helpful advice and revision of the manuscript.

\section{References}

1. Douillard JY, Hoff PM, Skillings JR, Eisenberg P, Davidson N, Harper P, Vincent MD, Lembersky BC, Thompson S, Maniero A and Benner SE: Multicenter phase III study of uracil/tegafur and oral leucovorin versus fluorouracil and leucovorin in patients with previously untreated metastatic colorectal cancer. J Clin Oncol 20: 3605-3616, 2002.

2. Carmichael J, Popiela T, Radstone D, Falk S, Borner M, Oza A, Skovsgaard T, Munier S and Martin C: Randomized comparative study of tegafur/uracil and oral leucovorin versus parenteral fluorouracil and leucovorin in patients with previously untreated metastatic colorectal cancer. J Clin Oncol 20: 3617-3627, 2002.

3. Lembersky BC, Wieand HS, Petrelli NJ, O'Connell MJ, Colangelo LH, Smith RE, Seay TE, Giguere JK, Marshall ME, Jacobs AD, Colman LK, Soran A, Yothers G and Wolmark N: Oral uracil and tegafur plus leucovorin compared with intravenous fluorouracil and leucovorin in stage II and III carcinoma of the colon: results from National Surgical Adjuvant Breast and Bowel Project Protocol C-06. J Clin Oncol 24: 2059-2064, 2006.

4. Shirasaka T, Shimamato Y, Ohshimo H, Yamaguchi M, Kato T, Yonekura K and Fukushima M: Development of a novel form of an oral 5-fluorouracil derivative (S-1) directed to the potentiation of the tumor selective cytotoxicity of 5-fluorouracil by two biochemical modulators. Anticancer Drugs 7: 548-557, 1996.

5. Ohtsu A, Baba H, Sakata Y, Mitachi Y, Horikoshi N, Sugimachi K and Taguchi T: Phase II study of S-1, a novel oral fluorophyrimidine derivative, in patients with metastatic colorectal carcinoma. S-1 Cooperative Colorectal Carcinoma Study Group. Br J Cancer 83: 141-145, 2000.

6. Longley DB, Harkin DP and Johnston PG: 5-fluorouracil: mechanisms of action and clinical strategies. Nat Rev Cancer 3: 330-338, 2003.

7. Ichikawa W, Uetake H, Shirota Y, Yamada H, Nishi N, Nihei Z, Sugihara K and Hirayama R: Combination of dihydropyrimidine dehydrogenase and thymidylate synthase gene expressions in primary tumors as predictive parameters for the efficacy of fluoropyrimidine-based chemotherapy for metastatic colorectal cancer. Clin Cancer Res 9: 786-791, 2003.

8. Fujii R, Seshimo A and Kameoka S: Relationships between the expression of thymidylate synthase, dihydropyrimidine dehydrogenase, and orotate phosphoribosyltransferase and cell proliferative activity and 5-fluorouracil sensitivity in colorectal carcinoma. Int J Clin Oncol 8: 72-78, 2003.

9. Ichikawa W, Uetake H, Shirota Y, Yamada H, Takahashi T, Nihei Z, Sugihara K, Sasaki Y and Hirayama R: Both gene expression for orotate phosphoribosyltransferase and its ratio to dihydropyrimidine dehydrogenase influence outcome following fluoropyrimidine-based chemotherapy for metastatic colorectal cancer. Br J Cancer 89: 1486-1492, 2003.

10. Radparvar S, Houghton PJ and Houghton JA: Effect of polyglutamylation of 5,10-methylenetetrahydrofolate on the binding of 5-fluoro-2'-deoxyuridylate to thymidylate synthase purified from a human colon adenocarcinoma xenograft. Biochem Pharmacol 38: 335-342, 1989.

11. Sakamoto E, Tsukioka S, Oie S, Kobunai T, Tsujimoto H, Sakamoto K, Okayama Y, Sugimoto Y, Oka T and Fukushima M: Folylpolyglutamate synthase and gamma-glutamyl hydrolase regulate leucovorin-enhanced 5-fluorouracil anticancer activity. Biochem Biophys Res Commun 365: 801-807, 2008.

12. Sadahiro S, Suzuki T, Maeda Y, Tanaka A, Ogoshi K, Kamijo A, Murayama C, Tsukioka S, Sakamoto E, Fukui Y and Oka T: Molecular determinants of folate levels after leucovorin administration in colorectal cancer. Cancer Chemother Pharmacol 65: 1198-1207, 2009.

13. Spears CP and Gustavsson BG: Methods for thymidylate synthase pharmacodynamics: serial biopsy, free and total TS, FdUMP and dUMP, and $\mathrm{H}_{4}$ PteGlu and $\mathrm{CH}_{2}-\mathrm{H}_{4}$ PteGlu assays. Adv Exp Med Biol 244: 97-106, 1988. 
14. Taomoto J, Yoshida K, Wada Y, Tanabe K, Konishi K, Tahara H and Fukushima M: Overexpression of the orotate phosphoribosyl-transferase gene enhances the effect of 5-fluorouracil on gastric cancer cell lines. Oncology 70: 458-464, 2006.

15. Priest DG, Schmitz JC, Bunni MA and Stuart RK: Pharmacokinetics of leucovorin metabolites in human plasma as a function of dose administered orally and intravenously. J Natl Cancer Inst 83: 1806-1812, 1991.

16. Schmitz JC, Grindey GB, Schultz RM and Priest DG: Impact of dietary folic acid on reduced folates in mouse plasma and tissues. Relationship to dideazatetrahydrofolate sensitivity. Biochem Pharmacol 48: 319-325, 1994.

17. Tsujimoto H, Tsukioka S, Koizumi K, Nakagawa F, Uchida J, Sugimoto Y, Oka T, Fukushima M and Watanabe T: Benefits of TS-1 plus leucovorin combination therapy for colorectal cancer: evaluation of therapeutic effect of TS-1 and leucovorin combination therapy using rodent model fed a low folate diet. Gan To Kagaku Ryoho 34: 413-418, 2007.
18. Tsukioka S, Uchida J, Tsujimoto H, Nakagawa F, Sugimoto Y, Oka T and Kiniwa M: Oral fluoropyrimidine S-1 combined with leucovorin is a promising therapy for colorectal cancer: Evidence from a xenograft model of folate-depleted mice. Mol Med Rep 2: 393-398, 2009.

19. Cheradame S, Etienne MC, Chazal M, Guillot T, Fischel JL, Formento P and Milano G: Relevance of tumoral folylpolyglutamate synthetase and reduced folates for optimal 5-fluorouracil efficacy: experimental data. Eur J Cancer 33: 950-959, 1997.

20. Sohn KJ, Smirnakis F, Moskovitz DN, Novakovic P, Yates Z, Lucock M, Croxford R and Kim YI: Effects of folylpolyglutamate synthetase modulation on chemosensitivity of colon cancer cells to 5-fluorouracil and methotrexate. Gut 53: 1825-1831, 2004. 